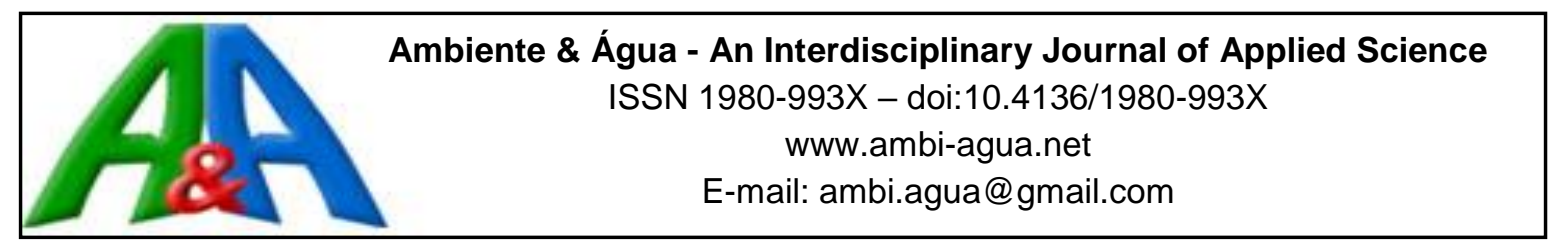

\title{
Impactos do uso do solo nos recursos hídricos da bacia de contribuição do Lago dos Manacás, Minas Gerais, Brasil
}

\author{
doi:10.4136/ambi-agua.1882
}

Received: 07 Mar. 2016; Accepted: 08 Jul. 2016

\author{
Cézar Henrique Barra Rocha*; Bruna Helena Coelho Pereira; \\ André Felipe Rocha da Silva; Márcio de Oliveira; \\ Antoine Philippe Casquin; Marinna Reis de Figueiredo

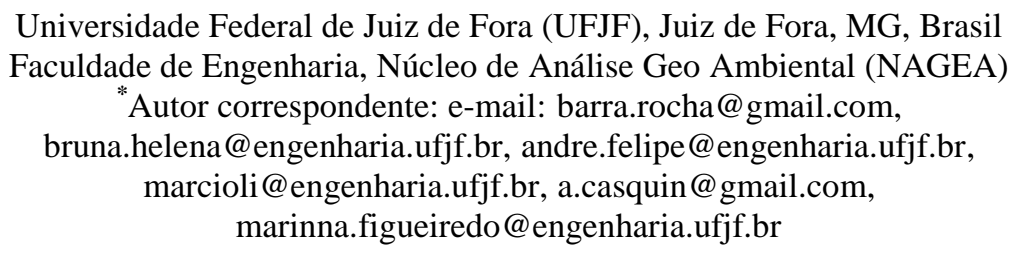

\section{RESUMO}

O Campus da Universidade Federal de Juiz de Fora, com 134,68 ha, está inserido em área urbana com grande crescimento imobiliário e com uma população de 27.600 usuários, acarretando a perda de áreas verdes, aumentando a geração de resíduos e efluentes. O objetivo desse artigo foi avaliar os impactos das atividades do Campus da UFJF na qualidade da água da Bacia de Contribuição do Lago dos Manacás (BCLM). Foram escolhidos cinco pontos para o monitoramento, considerando uma nascente (P1) e as fozes dos quatro córregos que deságuam no Lago dos Manacás (P2 a P5). As amostras foram coletadas mensalmente, durante o período de novembro de 2013 a outubro de 2014. Foram medidos pH, Oxigênio Dissolvido (OD), Demanda Química de Oxigênio (DQO), Condutividade Específica e Turbidez. Os resultados mostraram que o OD aumenta da nascente de um córrego (P1) para sua foz (P2) com valores baixos nas demais fozes, destacando uma mediana de $1,3 \mathrm{mg} \mathrm{L}^{-1}$ no P5; a DQO não segue um padrão, ficando com as medianas maiores que $6 \mathrm{mg} \mathrm{O}_{2} \mathrm{~L}^{-1} \mathrm{em}$ todos os pontos, com destaque para o $\mathrm{P} 4 \operatorname{com} 25,5 \mathrm{mg} \mathrm{O}_{2} \mathrm{~L}^{-1}$, sub-bacia na qual estão localizados a maioria dos laboratórios da universidade; a Turbidez ficou pior no P5 com 39,42 UNT, coincidindo com a sub-bacia mais afetada por obras e movimentação de terra; a Condutividade Específica aumentou gradativamente de $\mathrm{P} 1$ até $\mathrm{P} 2$, não diferenciando estatisticamente as medianas entre P3, P4 e P5. O Lago dos Manacás enquadra-se na Classe 2 e, de acordo com a Resolução CONAMA no 357/2005, há uma desconformidade entre os padrões estabelecidos e os resultados encontrados, com destaque para o OD nas seções 4 e 5 , com valores atuais compatíveis com a Classe 4 de frequente anaerobiose.

Palavras-chave: gestão de recursos hídricos, monitoramento ambiental, UFJF.

\section{Land use impacts on water resources of the Manacás Lake Basin, Minas Gerais, Brazil}

\section{ABSTRACT}

The 134.68 ha campus of the Federal University of Juiz de Fora (UFJF) is in urban area impacted by new developments. The population of 27,600 consumers causes the loss of green 
areas and generates increasing waste and sewage water. This study evaluated the impact of UFJF Campus activities on water quality, emphasizing the Manacás Lake Basin (MLB). The evaluation was based on five monitoring points: a spring (P1) and the four mouths of the streams that flow into Manacás Lake (P2 to P5). Water samples were collected monthly between November 2013 and October 2014, and pH, DO, COD, Conductivity and Turbidity were analyzed. The results showed that the DO increases from the spring (P1) to the mouth (P2), with low values at the other points, and a median of $1.3 \mathrm{mg} \mathrm{L}^{-1}$ at P5. COD does not follow a pattern, registering medians higher than $6 \mathrm{mgO}_{2} \mathrm{~L}^{-1}$ at all points, highlighting $\mathrm{P} 4$ with $25.5 \mathrm{mgO}_{2} \mathrm{~L}^{-1}$, the sub-basin which contains most of the Labs. Worse turbidity was found at P5, with 39.42 UNT, coinciding with the sub-basin most affected by construction. Conductivity gradually increased from $\mathrm{P} 1$ to $\mathrm{P} 2$, and medians were not statically different for P3, P4, and P5. Manacá Lake is Class 2 according to CONAMA resolution $\mathrm{N}^{\circ} 357 / 2005$, but the results showed that the DO at P4 and P5 has values of Class 4 water, with frequent anaerobiosis.

Keywords: environmental monitoring, UFJF, water resources management.

\section{INTRODUÇÃO}

A importância de se refletir sobre o gerenciamento dos recursos hídricos no Brasil vem crescendo frente à crise hídrica enfrentada nos últimos anos. Os ecossistemas de água doce, em termos de quantidade e qualidade, são os mais degradados em esfera global. Dentre esses ecossistemas estão os lagos e rios, que apesar de representar uma pequena parcela da água total do planeta, são de grande relevância em relação à água potável acessível (Gleick et al., 2014; OECD, 2015).

Em todo o mundo, lagos e rios vêm sendo alvos de monitoramento, análises e pesquisas, a fim de conhecer os impactos que sofrem. Dentre eles, podem-se citar o aumento de salinidade, alterações de temperatura e contaminação por componentes químicos industriais e agrícolas. O monitoramento da qualidade da água se faz através do acompanhamento periódico dos parâmetros físico-químicos, e fornece subsídios para avaliar as condições do recurso hídrico para tomada de decisões no gerenciamento do corpo d'água (Akbar et al., 2011; Bhattacharyya e Kapil, 2010; Marotta et al., 2008; Rocha et al., 2014; Rocha e Costa, 2015; Sheela et al., 2012).

O Lago dos Manacás é um lago artificial com um espelho de 1,37 ha. Está inserido no Campus sede da Universidade Federal de Juiz de Fora (UFJF), o qual possui uma área total de 134,68 ha. A Bacia de Contribuição do Lago dos Manacás (BCLM) tem área de 82,85 ha (Figura 1), compondo as sub-bacias contribuintes do córrego Independência, que deságua no rio Paraibuna, que por sua vez faz parte da Bacia Hidrográfica do rio Paraíba do Sul. De acordo com a Deliberação Normativa nº16/1996 do Conselho Estadual de Política Ambiental (COPAM) (Minas Gerais, 1996), a BCLM e o córrego Independência são enquadrados na Classe 2, devido à localização de sua foz em trecho do rio Paraibuna, que é enquadrado dessa forma.

Após a adesão ao Programa de Apoio a Planos de Reestruturação e Expansão das Universidades Federais (REUNI), implementado a partir do ano de 2009, verificou-se um aumento da área construída no Campus da UFJF e da população usuária deste espaço. Oliveira (2008) estimou que, após o REUNI, mais de 27.600 circulariam pelo campus sede da UFJF atualmente, o que corresponde a uma população maior do que $91 \%$ dos 142 municípios da Zona da Mata mineira, região onde está inserida a Instituição. Para chegar a essa estimativa foram considerados os números anteriores ao REUNI e as expansões previstas, somando-se aos docentes, discentes e técnicos administrativos, o grande número de trabalhadores terceirizados, prestadores de serviços, concessionários, empresas incubadas, os usuários dos 
serviços comunitários, e ainda, os visitantes que utilizam a área do campus para exercícios físicos e lazer. Com a expansão houve a perda de áreas verdes para implantação de estacionamentos e prédios com consequente impermeabilização do solo, aumentando assim o escoamento superficial e, desse modo, os impactos diretos e indiretos sobre os recursos hídricos (Yu et al., 2013). Além disso, o aumento das atividades acadêmicas acarreta em maior uso dos laboratórios, gerando ruídos, resíduos e principalmente efluentes que chegam no Lago dos Manacás situado no nível de base da Bacia do Campus da UFJF, conforme Figura 1.

Buscando harmonizar todas essas mudanças na estrutura física da UFJF com as dimensões ambientais e sociais, tem-se a necessidade do planejamento e implantação de medidas efetivas que visem o compromisso institucional com a sustentabilidade (Oliveira, 2009). Pesquisas vêm sendo realizadas no Campus da UFJF com vistas ao monitoramento dos impactos causados pelas atividades rotineiras, destacando o tráfego de veículos, o lançamento de esgotos domésticos, efluentes de laboratórios e o grande volume de obras. Estudos limnológicos iniciados por Azevedo et al. (2003) descobriram uma nova espécie de cianobactéria (Limnothrix bicudoi) no Lago dos Manacás. Posteriormente, Soares et al. (2009) estudaram as fases de equilíbrio desta espécie, observando que a abundância é influenciada pela temperatura e pelo período sazonal.

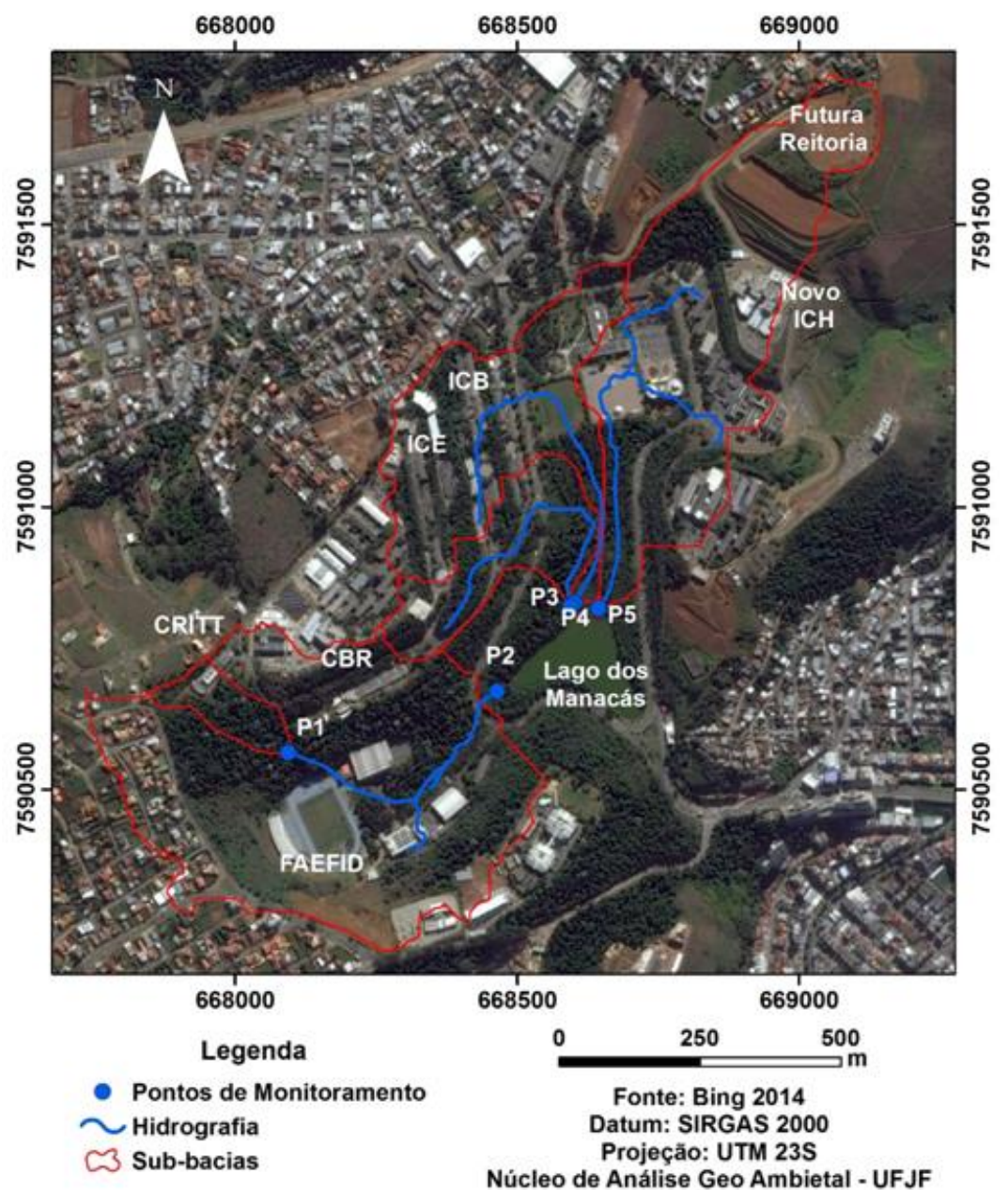

Figura 1. Carta da Bacia de Contribuição do Lago dos Manacás BCLM.

A fauna bentônica do lago foi estudada por alguns pesquisadores, destacando-se a 
pesquisa sobre decomposição de folhas por larvas de Chironomidae (Silveira et al., 2013) e o estudo realizado por Martins (2011), o qual analisou a decomposição de macrófitas por oligochaeta (Annelida: Clitellata). Também Frizzera (2011) desenvolveu trabalho sobre o tema e, como conclusões importantes, verificou que a alteração das condições das variáveis ambientais durante o período de coleta (2009-2010) contribuiu para alteração na estrutura da comunidade de Chironomidae e Oligochaeta e que, através da análise da assembléia dos grupos Chironomidae e Oligochaeta e das variáveis abióticas, foi possível verificar que o Lago dos Manacás apresentou alteração da qualidade de suas águas durante o período de estudo.

Marques et al. (2014) realizaram um estudo da qualidade das águas do Campus da UFJF, tendo como objeto de estudo o Lago dos Manacás (nível de base da sub-bacia do Campus), onde foi estipulada a quantidade de esgoto produzida diariamente no Campus. Já Oliveira et al. (2013) tiveram seu estudo voltado para a qualidade ambiental das 28 nascentes existentes no Campus da UFJF, onde verificaram um grau de impacto ambiental considerado médio, com $44,5 \%$ razoável e $40,7 \%$ na classe ruim, incluindo a nascente P1 utilizada nessa pesquisa com resultados de coliformes fecais de $1200 \mathrm{UFC} / 100 \mathrm{~mL}$.

Considerando os estudos já realizados e a importância da gestão dos recursos hídricos, o objetivo desse trabalho foi avaliar os impactos das atividades do Campus da UFJF na qualidade da água da Bacia de Contribuição do Lago dos Manacás.

\section{MATERIAL E MÉTODOS}

O Campus da Universidade Federal de Juiz de Fora se localiza na região oeste da cidade de Juiz de Fora e sua área total é de 1.346.793,80 m² (UFJF, 2015).

Foram escolhidos cinco pontos para o monitoramento da qualidade da água, partindo de uma nascente dentro de fragmento de Mata Atlântica entre o Centro de Biologia da Reprodução (CBR) e a Faculdade de Educação Física (FAEFID), denominado P1, e a foz dos principais córregos que cortam o Campus: P2 - Foz do córrego da FAEFID; P3, P4 e P5 - Foz de córrego a direita, meio e esquerda do Lago dos Manacás, respectivamente. Com o uso do software ArcGIS 10.2 foi gerado o Modelo Digital de Elevação Hidrograficamente Condicionado (MDEHC) com resolução de um metro, através das curvas de níveis obtidas pelo LiDAR e disponibilizadas pela Prefeitura de Juiz de Fora (Juiz de Fora, 2007). A partir do MDEHC, foi produzida uma Carta com destaque para as sub-bacias dos pontos de monitoramento, hidrografia e relevo (Figura 2).

As amostras de água foram coletadas mensalmente durante o período de novembro de 2013 a outubro de 2014, totalizando 12 meses, abrangendo um período de seca e outro de chuva, completando um ciclo hidrológico. Segundo o Plano Diretor de Desenvolvimento Urbano (Juiz de Fora, 2000) na cidade de Juiz de Fora há uma sazonalidade no clima bem definida, sendo a estação chuvosa de outubro a abril, com temperaturas mais altas e maiores precipitações pluviométricas, e a estação seca de maio a setembro, período mais frio e com menor incidência de chuvas.

Adotaram-se medidas e procedimentos para a realização das coletas e conservação das amostras, conforme estabelecido no Guia Nacional de Coleta e Preservação de Amostras (ANA, 2011).

Foram medidos parâmetros físico-químicos em campo utilizando a Sonda Multiparamétrica Modelo Hanna HI 9828, além das análises em bancada. Os parâmetros medidos diretamente pela sonda foram $\mathrm{pH}$, Condutividade Específica e Oxigênio Dissolvido (OD). A Turbidez foi aferida em bancada através de Turbidímetro Hanna modelo HI 93703. A Demanda Química de Oxigênio (DQO) foi obtida por oxidação por dicromato seguindo metodologia preconizada pela APHA (2012). A escolha desses parâmetros se deu em função 
dos equipamentos disponíveis (devidamente calibrados), dos resultados obtidos com relação ao observado em campo, além da comparação com trabalhos de outros pesquisadores.

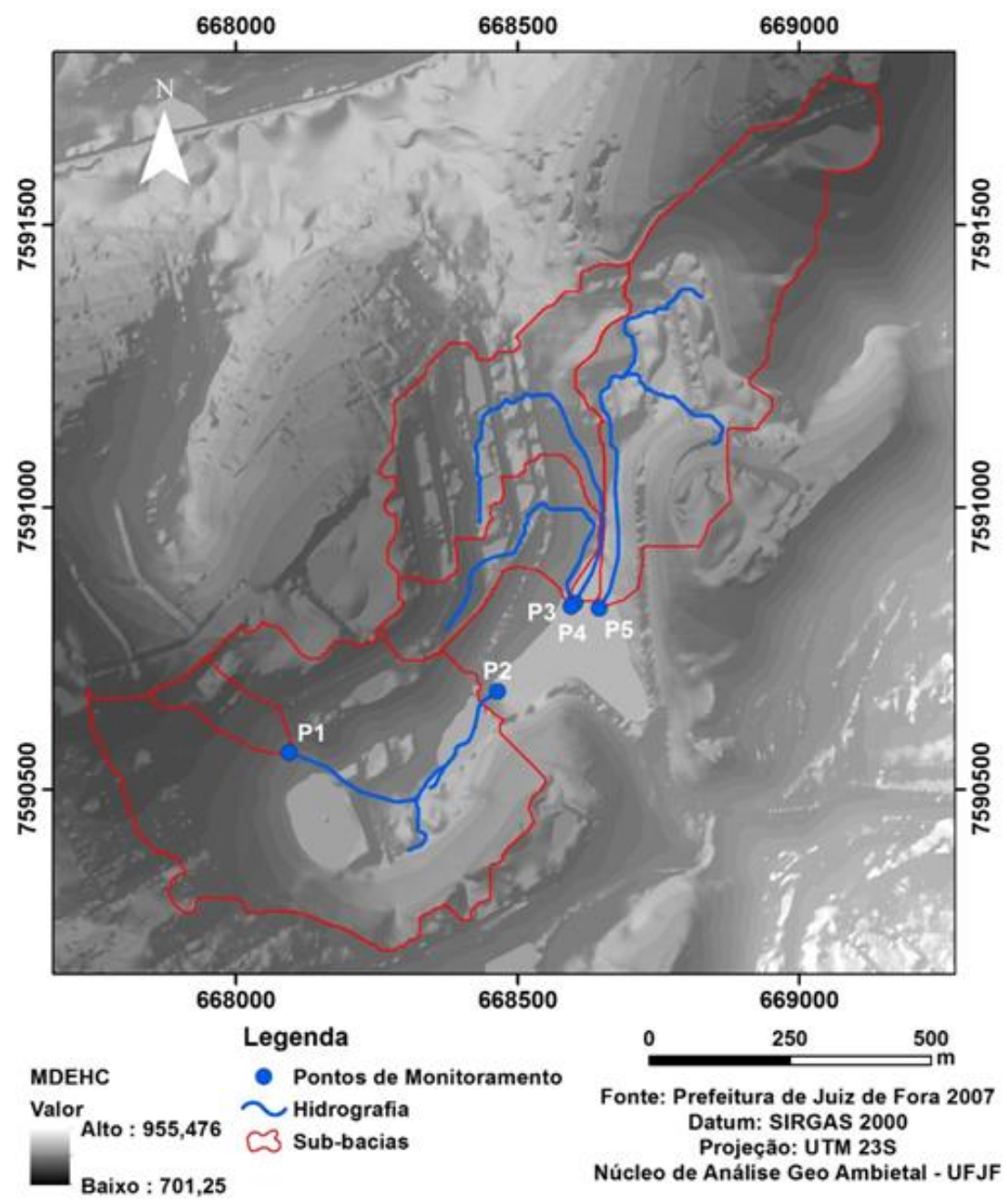

Figura 2. Modelo Digital de Elevação Hidrograficamente Condicionado da BCLM.

Os dados obtidos foram tabulados em uma planilha do Microsoft Office Excel 2010, onde foram gerados valores de média e desvio padrão para cada parâmetro nos períodos de seca e chuva. Os dados também foram importados para o programa PAST - Palaeontological Statistics, no qual foram gerados diagramas Boxplot, obtendo as medianas e os valores máximos e mínimos para os parâmetros de cada ponto.

Devido à natureza não normal de dados de qualidade da água (Helsel, 1987), foi utilizado o teste não-paramétrico de Mann-Whitney para verificação de diferenças significativas ou muito significativas entre os pontos amostrados $(p<0,05)$ e $(p<0,01)$, respectivamente. Os pontos P1 e P2 representam a nascente e a foz do mesmo córrego, assim, verificar se houve diferença significativa entre esses pontos levaria a uma análise dos processos ao longo do curso d'água. Quanto aos pontos P3, P4 e P5, como se tratam de córregos distintos com suas respectivas sub-bacias, tal verificação ajudaria na investigação de como o uso e cobertura do solo em cada área interferiu na qualidade da água conforme a Figura 1.

A fim de se conferir a adequação desse recurso hídrico aos padrões da legislação ambiental, os valores encontrados para os parâmetros Turbidez, $\mathrm{pH}$ e OD foram comparados com os limites estabelecidos pela Resolução n³57 de 2005 do Conselho Nacional de Meio 
Ambiente - CONAMA (Brasil, 2005) para um curso d'água de Classe 2.

\section{RESULTADOS E DISCUSSÃO}

\subsection{Potencial hidrogeniônico (pH)}

A Resolução CONAMA 357/2005, ao definir padrões de qualidade da água, rege que para as águas de classe 2, o pH deve ter valores entre 6,0 e 9,0 (Brasil, 2005). Como se observa na Figura 3, o ponto P1 apresentou pH ácido, ao redor de 6,1. Tal resultado pode ser atribuído ao tipo de solo presente na região, o latossolo vermelho-amarelo (Sousa e Lobato, 2015), uma vez que a dissolução de rochas é um dos fatores que influenciam o pH em nascentes. Além disso, a água da nascente apresenta-se protegida por um fragmento de Mata Atlântica (Figura 1), o que oferece proteção quanto a erosão, lixiviação e consequentes assoreamento e carreamento de nutrientes. Já na seção seguinte (P2) o pH é maior, aproximando-se do valor neutro, não ocorrendo grandes variações nos valores observados. Comparando as 5 seções do estudo, a maior diferença observada foi entre os pontos P1 e P2, com medianas 6,09 e 6,63 respectivamente, permanecendo, contudo, ácido (Figura 3). Tal diferença pode ser comprovada através do teste de Mann-Whitney, sendo que nestes pontos encontrou-se o valor de $\mathrm{p}=0,0216$. Não houve diferenças significativas dos valores de $\mathrm{pH}$ entre os demais pontos. Esse resultado pode indicar que o uso e cobertura do solo nas subbacias de contribuição pouco interferiu na variação do $\mathrm{pH}$ dos córregos, sendo que a influência do latossolo vermelho-amarelo pode ter prevalecido, sendo ainda mais representativa na nascente (P1) (Donadio et al., 2005), e as medianas dos cinco pontos ficaram abaixo de 7,0, porém acima de 6,0, respeitando assim os limites da Resolução CONAMA no $357 / 2005$.

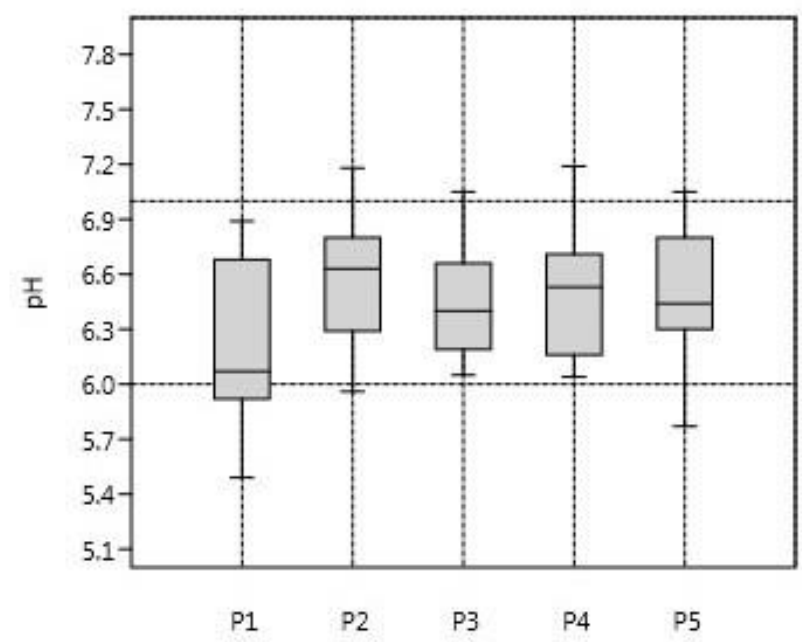

Figura 3. Boxplot do parâmetro $\mathrm{pH}$ nos cinco pontos de monitoramento da BCLM.

\subsection{Oxigênio Dissolvido (OD)}

Na Figura 4 verifica-se que a concentração de OD aumentou do P1 para o P2, o que pode ser entendido pela oxigenação do curso d'água no percurso entre a nascente $(\mathrm{P} 1)$ e sua foz (P2). Nos demais pontos os valores foram menores, com o pior resultado no P5 com mediana de $1,3 \mathrm{mg} \mathrm{L}^{-1}$. Nos pontos P4 e P5 foram encontradas condições de anaerobiose em alguns meses. A Resolução CONAMA n ${ }^{\circ} 357 / 2005$ define que o valor mínimo para este parâmetro deve ser de $5 \mathrm{mg} \mathrm{L}^{-1}$ de OD (Brasil, 2005). As medianas de todos os pontos não atenderam a este limite. 
Segundo Von Sperling (2005), valores de OD inferiores à saturação são indicativos da presença de matéria orgânica, provavelmente de esgoto. As sub-bacias de contribuição dos pontos P4 e P5 são as que possuem maior quantidade de instalações acadêmicas (destacando os laboratórios) e administrativas, como evidenciado na Figura 1. Isso sugere também que essas áreas concentram maior geração de esgoto sanitário. Como todas as áreas das bacias de drenagem dos pontos estudados se encontram dentro dos limites do Campus da UFJF, a interferência de fontes externas de poluição pode ser descartada. Assim, devido à idade das instalações sanitárias do campus, iniciadas na década de 1960, possíveis falhas na rede de coleta de esgoto podem estar contaminando os recursos hídricos. As bactérias que degradam a matéria orgânica são organismos aquáticos que consomem OD, explicando os baixos valores encontrados. E ainda, o campus é uma área urbanizada que não possui fauna terrestre expressiva que possa contribuir com a carga orgânica nos cursos d'água. A degradação da matéria orgânica consome o oxigênio dissolvido na água através da ação catalisadora de inúmeros microrganismos, entre os quais predominam as bactérias aeróbias (Branco, 1986).

A análise do teste de Mann-Whitney apontou que houve diferença significativa entre P2 e P3 com $\mathrm{p}=0,04036$. E que os pontos P4 e P5, os quais apresentaram as menores concentrações de OD, chegam a apresentar diferença muito significativa de P1 e P2 com $\mathrm{p}=0,001215$. Esses resultados reforçam a possível influência de esgoto nos valores de OD para os pontos $\mathrm{P} 4$ e P5.

Os teores de OD do P2 ao P5 são baixos considerando-se córregos de cabeceira. Estudo realizado em córrego de primeira ordem numa reserva biológica no mesmo município, Rosa et al., (2011) encontrou o OD atingindo o valor de saturação. No mesmo bioma em outros estados brasileiros, considerando córregos de primeira e segunda ordem, Cunha et al. (2014) acharam valores de OD entre $5 \mathrm{mg} \mathrm{L}^{-1}$ e $6 \mathrm{mg} \mathrm{L}^{-1}$, e Suriano et al. (2011), valores entre 7,85 $\mathrm{mg} \mathrm{L}^{-1}$ e $10,8 \mathrm{mg} \mathrm{L}^{-1}$ em ambientes preservados.

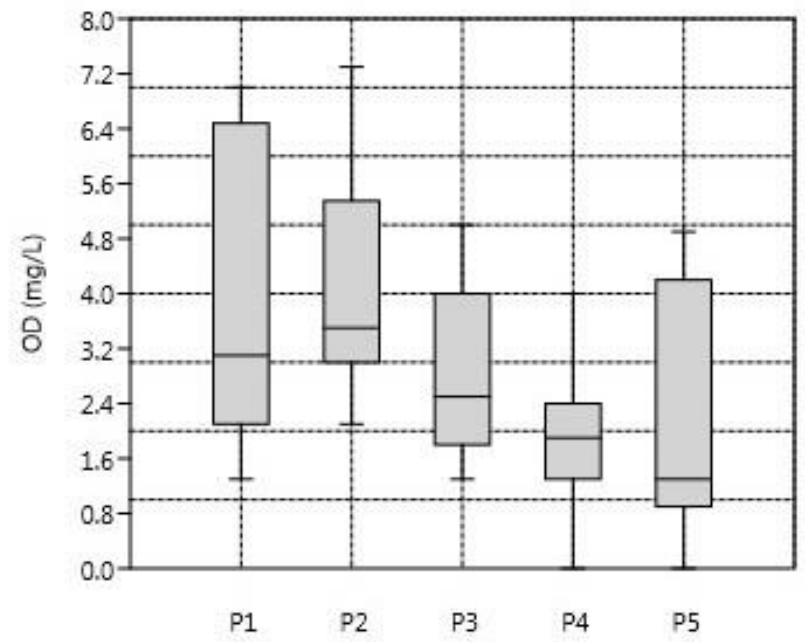

Figura 4. Boxplot do parâmetro OD nos cinco pontos de monitoramento da BCLM.

\subsection{Demanda Química de Oxigênio (DQO)}

A Resolução CONAMA n ${ }^{\circ}$ 357/2005 não define limites para este parâmetro (Brasil, 2005). Seu uso na gestão das águas geralmente se dá em associação com a DBO (Demanda Bioquímica de Oxigênio) para o controle de sistemas de tratamento de efluentes (CETESB, 2009). No entanto, a comparação dos valores de DQO entre os pontos desta pesquisa auxilia na diferenciação entre eles e na indicação de possível poluição por esgotos. Na Figura 5, observa-se que a DQO não demonstrou um comportamento definido. Seus valores oscilaram 
entre os pontos, sendo que a mediana obteve maior diferença entre P1 e P4, sendo seus valores, respectivamente, $6 \mathrm{mgO}_{2} \mathrm{~L}^{-1}$ e $25,5 \mathrm{mgO}_{2} \mathrm{~L}^{-1}$, o que pode ser verificado pelo teste de Mann-Whitney com $\mathrm{p}=0,04285$.

O maior valor de DQO no ponto P4 sugere possível falha nas redes de efluentes do Instituto de Ciências Exatas (ICE) e do Instituto de Ciências Biológicas (ICB), onde se concentram os laboratórios dos cursos de Física, Química e Biologia, além dos esgotos sanitários (Figura 1). Como já relatado, não há fontes externas a serem consideradas. Nesses laboratórios são gerados efluentes nas atividades de ensino e pesquisa. Mesmo considerando que a UFJF adota um programa de descarte de resíduos perigosos, a lavagem das vidrarias e outros instrumentos e materiais gera efluentes líquidos contaminados. Os efluentes de laboratórios possuem uma DQO particularmente alta. Alves et al. (2005) mediram uma DQO de $1350 \mathrm{mg} \mathrm{L}^{-1}$ para um efluente de laboratório de controle da poluição, similar a muitos laboratórios instalados na bacia de drenagem do P4. Uma vez que o campus não dispõe de sistema de tratamento de efluentes, estes são coletados e enviados para a rede pública municipal. Possíveis vazamentos na rede de coleta podem levar à poluição dos córregos e, por consequência, do Lago dos Manacás.

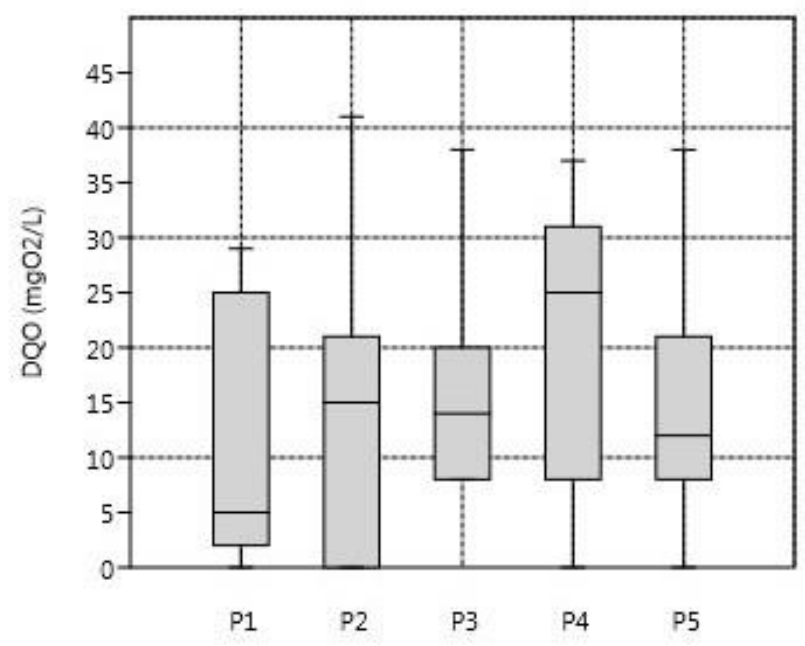

Figura 5. Boxplot do parâmetro DQO nos cinco pontos de monitoramento da BCLM.

\subsection{Turbidez}

Observa-se na Figura 6 uma mediana baixa em P2 com valor de 1,57 UNT, o que pode ser justificado pela presença de matas ciliares no ponto analisado (Figura 1). Segundo Rocha e Costa (2015), essas faixas (matas) reduzem o impacto de fontes de poluição através do efeito "filtro", destacando a retenção de sedimentos finos, matéria orgânica, produtos tóxicos, diminuindo os processos de assoreamento do corpo d'água, evitando a erosão e estabilizando os solos marginais, se comportando como uma verdadeira barreira física.

Nos demais pontos, os valores foram muito variáveis, com o valor máximo atingindo 39,42 UNT em P5. Para águas de classe 2 a Resolução CONAMA nº 357/2005 define que limite para a Turbidez é de 100 UNT (Brasil, 2005). Deste modo, os valores encontrados não infringem tal norma. No entanto, a comparação deste parâmetro entre os pontos evidencia as consequências da interferência antrópica. Essa seção, P5, recebeu os impactos dos maiores volumes de obras no Campus, destacando a construção do novo prédio do Instituto de Ciências Humanas (ICH) no início da porção Nordeste e a terraplanagem de dois patamares para instalação da futura Reitoria da UFJF no limite da porção Nordeste (Figura 1). Obras e movimentação de terra numa bacia favorecem a ocorrência de processos erosivos e o 
consequente carreamento de partículas de solo para os cursos d'água, provocando assim o aumento dos sólidos em suspensão e da Turbidez do corpo hídrico. Esse aumento da Turbidez é uma ameaça para o ecossistema segundo Wantzen e Mol (2013), e não foram adotadas medidas preventivas como recomendado e detalhado por Harbor (1999) durante as obras.

A variação dos resultados deste parâmetro entre os pontos pode ser confirmada pela análise do teste de Mann-Whitney, onde foram indicadas diferenças muito significativas entre todos os pontos, com exceção entre P1 e P2 que obteve somente diferença significativa com $\mathrm{p}=0,0258$, e entre P3 e P4 sem diferença significativa com $\mathrm{p}=0,7289$.

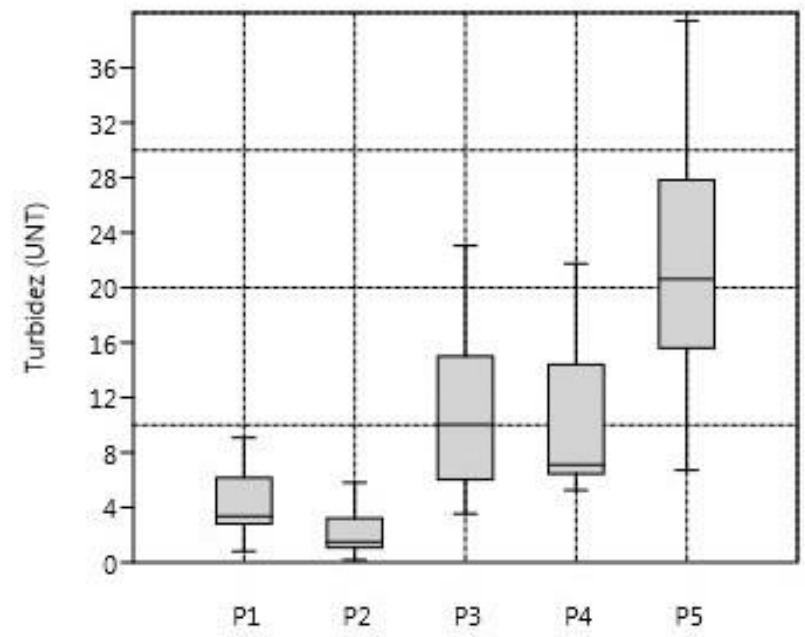

Figura 6. Boxplot do parâmetro Turbidez nos cinco pontos de monitoramento da BCLM.

\subsection{Condutividade Específica}

A Resolução CONAMA n ${ }^{\circ}$ 357/2005 não define limites para este parâmetro (Brasil,

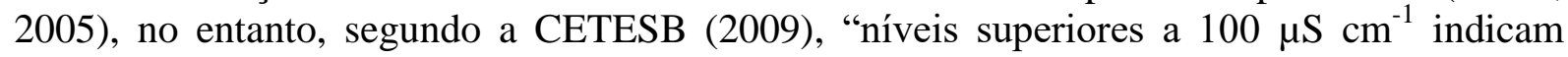
ambientes impactados".

Neste estudo não foram verificados valores de condutividade específica superiores a $100 \mu \mathrm{S} \mathrm{cm}^{-1}$. A condutividade específica é um parâmetro fácil de ser medido e apresenta numerosas correlações com variáveis de qualidade da água (Wang e Yin, 1997), explicando sua medição em estudos de monitoramento de qualidade da água. A Figura 7 ilustra a variação dos valores encontrados para a condutividade específica, a qual apresentou aumento de P1, com mediana de $14,36 \mu \mathrm{S} \mathrm{cm} \mathrm{cm}^{-1}$, para $\mathrm{P} 2$, com mediana de $30,47 \mu \mathrm{S} \mathrm{cm} \mathrm{cm}^{-1}$. Essa diferença entre a nascente e a foz do mesmo córrego pode indicar processos de poluição difusa aumentando a quantidade de sólidos dissolvidos em P2, o que requer mais investigações visto que, para os demais parâmetros analisados, o ponto P2 apresentou melhores resultados em comparação a P1. O maior valor de condutividade específica e a maior mediana foram do ponto P5 com um valor de $54,21 \mu \mathrm{S} \mathrm{cm}^{-1}$. Mais uma vez sugere-se que processos erosivos resultantes da movimentação de terra na sub-bacia de contribuição do P5 estejam também influenciando a condutividade da água neste ponto, aumentando os íons dissolvidos na água.

Com o teste de Mann-Whitney verificou-se que não houve diferenças significativas entre os valores de condutividade específica para os pontos P3, P4 e P5 (P3-P4 p=0,7583; P3-P5 p=0,1741; P4-P5 p=0,4116). Verificou-se também que para P1 (nascente) e P2 (foz) há diferenças muito significativas em relação a todos os outros pontos (P1-P3, P1-P4 e P1-P5 $\mathrm{p} \leq 0,001 ; \mathrm{P} 2-\mathrm{P} 3, \mathrm{P} 2-\mathrm{P} 5 \mathrm{p} \leq 0,005 ; \mathrm{P} 2-\mathrm{P} 4 \mathrm{p}=0,0905$ devido à amplitude de variação do P4), sugerindo que a sub-bacia que engloba esses pontos do mesmo córrego (CRITT, CBR e 
FAEFID) apresenta melhores condições ambientais que as demais sub-bacias conforme as Figuras 1 e 7.

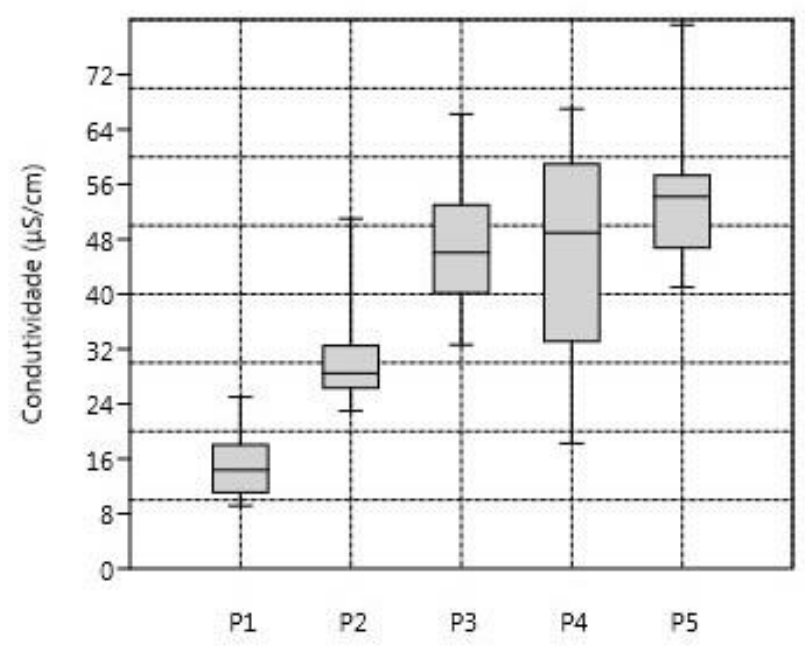

Figura 7. Boxplot do parâmetro Condutividade Específica nos cinco pontos de monitoramento da BCLM.

A Tabela 1 apresenta a comparação entre os padrões estabelecidos pela Resolução CONAMA no 357/2005 (Brasil, 2005) dos parâmetros de pH, OD e Turbidez para um curso d'água de Classe 2, com os valores obtidos no presente trabalho nos cinco pontos de monitoramento.

Tabela 1. Comparação entre os padrões estabelecidos pela Resolução CONAMA no 357/2005 (Brasil, 2005) com os valores encontrados nos pontos de monitoramento.

\begin{tabular}{|c|c|c|c|c|c|c|}
\hline & \multicolumn{2}{|l|}{$\mathrm{pH}$} & \multicolumn{2}{|c|}{$\mathrm{OD}\left(\mathrm{mg} \mathrm{L}^{-1}\right)$} & \multicolumn{2}{|c|}{ Turbidez (UNT) } \\
\hline \multicolumn{7}{|c|}{ Resolução CONAMA nº 357/2005 } \\
\hline & \multicolumn{2}{|c|}{6,0 a 9,0} & \multicolumn{2}{|c|}{$\geq 5$} & \multicolumn{2}{|c|}{$\leq 100$} \\
\hline & Seca & Chuva & Seca & Chuva & Seca & Chuva \\
\hline $\mathrm{P} 1$ & $6,31 \pm 0,36$ & $6,11 \pm 0,46$ & $3,93 \pm 1,47$ & $3,72 \pm 2,41$ & $3,26 \pm 0,31$ & $4,76 \pm 3,85$ \\
\hline $\mathrm{P} 2$ & $6,84 \pm 0,30$ & $6,45 \pm 0,31$ & $4,6 \pm 1,56$ & $3,89 \pm 1,72$ & $2,37 \pm 2,30$ & $1,98 \pm 1,49$ \\
\hline P3 & $6,64 \pm 0,28$ & $6,30 \pm 0,22$ & $2,73 \pm 0,51$ & $2,96 \pm 1,46$ & $17,56 \pm 6,18$ & $7,44 \pm 3,60$ \\
\hline P4 & $6,57 \pm 0,12$ & $6,46 \pm 0,42$ & $1,94 \pm 1,52$ & $2,06 \pm 0,77$ & $8,35 \pm 4,16$ & $12,88 \pm 6,06$ \\
\hline P5 & $6,66 \pm 0,26$ & $6,37 \pm 0,32$ & $1,42 \pm 1,04$ & $2,49 \pm 1,98$ & $18,12 \pm 8,83$ & $25,66 \pm 12,03$ \\
\hline
\end{tabular}

Em relação ao parâmetro OD, constatou-se a não conformidade de todos os valores com os limites, independente da seção de monitoramento e da sazonalidade. Os parâmetros pH e Turbidez encontraram-se dentro dos padrões estabelecidos para Classe 2 de 6,0 e 9,0 para pH, e até $100 \mathrm{UNT}$, respectivamente.

O ponto $\mathrm{P} 2$ apresentou os melhores resultados com relação aos parâmetros enquadrados. Como já registrado, essa seção é favorecida por uma mata ciliar que parece ter atenuado os impactos, provavelmente de esgotos e poluição difusa. 


\section{CONCLUSÕES}

A qualidade da água de uma determinada região é resultado, dentre outros fatores, da cobertura vegetal existente e da forma de uso do solo. O Campus da UFJF se expandiu com construção de novas instalações e aumento das atividades acadêmicas e científicas. Tal expansão, sem adequado gerenciamento dos recursos hídricos, resultou em impactos negativos nos corpos d'água, representados pelos baixos valores de OD em todas as seções de monitoramento. Os valores diferenciados de DQO e de Turbidez também chamam a atenção para as atividades antrópicas em algumas sub-bacias. Mesmo com o ritmo das obras no campus diminuindo, os impactos ainda são observados, contribuindo para que a qualidade da água no espaço monitorado esteja distante da Classe 2. Os resultados encontrados são compatíveis com a Classe 4, indicada apenas para navegação e harmonia paisagística.

A comunidade universitária e a alta administração da UFJF devem buscar uma política que vise uma maior proteção dos recursos hídricos da bacia de contribuição do Lago dos Manacás. É necessária a implementação de instrumentos que permitam maiores informações acerca das características desses recursos, como monitoramento dos corpos d'água, mapeamento e adequação das redes de esgoto, conjuntamente com soluções que conciliem o atendimento das necessidades institucionais relacionadas ao espaço físico com a conservação do meio ambiente.

Finalmente, com a escassez dos recursos hídricos e o aumento dos custos com abastecimento público, as águas do Lago dos Manacás poderiam ser utilizadas para abastecer o próprio Campus, gerando uma economia a ser revertida para a própria comunidade.

\section{AGRADECIMENTOS}

Agradecemos aos pesquisadores do PGECOL, do NAGEA e do LADINAA pelo apoio nos trabalhos de campo, laboratório e discussões, e à UFJF pelas bolsas cedidas.

\section{REFERÊNCIAS}

AKBAR, T. A.; HASSAN, Q. K.; ACHARI, G. A methodology for clustering lakes in Alberta on the basis of water quality parameters. Clean - Soil, Air, Water, v. 39, n. 10, p. 916-924, 2011. http://dx.doi.org/10.1002/clen.201100050

ALVES, L. C.; CAMMAROTA, M. C.; FRANÇA, F. P. Inibição de lodo biológico anaeróbio por constituintes de efluente de laboratório de controle de poluição. Engenharia sanitária e ambiental, v. 10, n. 3, p. 236-242, 2005. http://dx.doi.org/10.1590/S141341522005000300008

AGÊNCIA NACIONAL DE ÁGUAS - ANA (Brasil). Guia nacional de coleta e preservação de amostras: água, sedimento, comunidades aquáticas e efluentes líquidos. Brasília, 2011.

AMERICAN PUBLIC HEALTH ASSOCIATION - APHA. Standard Methods for the examination of water and wastewater. 22. ed. Washington D.C., 2012.

AZEVEDO, M. T. de P. et al. Limnthrix bicudoi, a new species of Cyanophyceae/ Cyanobacteria from Southeast of Brazil. Algological Studies, v. 109, p. 93-102, 2003. http://dx.doi.org/10.1127/1864-1318/2003/0109-0093 
BHATTACHARYYA, K. G.; KAPIL, N. Impact of urbanization on the quality of water in a naturalreservoir: a case study with the DeeporBeel in Guwahaticity, India. Water and Environment Journal, n. 24, p. 83-96, 2010. http://dx.doi.org/10.1111/j.17476593.2008.00157.x

BRANCO, S. M. Hidrobiologia aplicada à engenharia sanitária. São Paulo: CETESB, 1986. $640 \mathrm{p}$.

BRASIL. Resolução 357, de 17 de março de 2005. Diário Oficial [da] República Federativa do Brasil, Brasília, 17 mar. 2005. Disponível em: www.mma.gov.br/ port/conama/res/res 05/res35705.pdf. Acesso em: 13 abr. 2014.

COMPANHIA DE TECNOLOGIA DE SANEAMENTO AMBIENTAL - CETESB. Índice de qualidade das águas. São Paulo, 2009.

CUNHA, J. C. S.; BARROS FILHO, R. G.; SILVA, R. P.; SANTOS, I. G. A.; RODRIGUES, G. G. Benthic macrofauna and the limnological parameters of a first-order stream in Atlantic Forest of Brazilian Northeast. Acta Limnologica Brasiliensia, v.26, n.1, Rio Claro, S.P., Brazil, Jan./Mar. 2014.

http://dx.doi.org/10.1590/S2179-975X2014000100005

DONADIO, N. M. M.; GAlBIATTI, J. A.; PAUlA, R. C. de. Qualidade da água de nascentes com diferentes usos do solo na bacia hidrográfica do córrego rico, São Paulo, Brasil. Engenharia Agrícola, v. 25, n. 1, p. 115-125, 2005. http://dx.doi.org/10.1590/S0100-69162005000100013

FRIZZERA, G. L. Limnologia, distribuição e composição da assembléia de oligochaeta (annelida: clitellata) e larvas de chironomidae (díptera) do lago dos Manacás, município de Juiz de Fora, MG. 2011. Dissertação (Mestrado em Ecologia) - Instituto de Ciências Biológicas, Universidade Federal de Juiz de Fora, Juiz de Fora, 2011.

GLEICK, P. H. et al. The World's Water Volume 8: The Biennial Report on Freshwater Resources. [S.1.]: Island Press, 2014.

HARBOR, J. Engineering geomorphology at the cutting edge of land disturbance: Erosion and sediment control on construction sites. Geomorphology, v. 31, n. 1-4, p. 247-263, 1999. http://dx.doi.org/10.1016/S0169-555X(99)00107-5

HELSEL, D. R. Advantages of nonparametric procedures for analysis of water quality data. Hydrological Sciences Journal, v. 32, n. 2, p. 179-190, 1987. http://dx.doi.org/10.1080 /02626668709491176

JUIZ DE FORA. Lei $\mathbf{n}^{\circ} 9.811$ de 27 de junho de 2000. Institui o plano diretor de desenvolvimento urbano de Juiz de Fora. Disponível em: http://www.leismunicipais.com.br/legislacao-de-juiz-de-fora/1391368/lei-9811-2000juiz-de-fora-mg.html. Acesso em: 07 maio 2014.

JUIZ DE FORA. Prefeitura Municipal. Secretaria de Planejamento e Gestão. Base Cartográfica Digital de Juiz de Fora. Esteio, 2007.

MAROTTA, H.; SANTOS, R. O. dos; ENRICH-PRAST, A. Monitoramento limnológico: um instrumento para a conservação dos recursos hídricos no planejamento e na gestão urbano-ambientais. Ambiente \& sociedade, v. 11, n. 1, p. 67-79, 2008. http://dx.doi.org/10.1590/S1414-753X2008000100006 
MARQUES, V.; AMARAL, A; MIRANDA, C. MOURA, M. N; MACHADO, P. J. O. Estudo da qualidade das águas do Campus da UFJF. In: SIMPÓSIO DE RECURSOS HÍDRICOS DA BACIA DO RIO PARAÍBA DO SUL, 2., 2014, São José dos Campos. Documentos... São José dos Campos: AGEVAP, 2014.

MARTINS, R. T. Colonization by oligochaetes (Annelida: Clitellata) in decomposing leaves of Eichhornia azurea (SW.) Kunth (Pontederiaceae) in neotropical lentic system. International Journal of Limnology, v. 47, p. 339-346, 2011. http://dx.doi.org/ 10.1051/limn/2011053

MINAS GERAIS. Deliberação Normativa COPAM n 016, de 24 de Setembro de 1996. Diário do Executivo - Minas Gerais, 02 out. 1996. Disponível em http://www.siam.mg.gov.br/sla/download.pdf?idNorma=113. Acesso em: 10 de julho de 2015.

OECD. Governança dos recursos hídricos no Brasil. Paris: OECD Publishing, 2015.

OLIVEIRA, M. de. Gestão ambiental em instituições de ensino superior. 2008. 76f. Trabalho de Conclusão de Curso (Especialização em Análise Ambiental) - Faculdade de Engenharia, Universidade Federal de Juiz de Fora, Juiz de Fora, 2008.

OLIVEIRA, M. de. Universidade e Sustentabilidade: proposta de diretrizes e ações para uma universidade ambientalmente sustentável. 2009. 90f. Dissertação (Mestrado em Ecologia aplicada ao Manejo e a Conservação dos Recursos Naturais) - Instituto de Ciências Biológicas, Universidade Federal de Juiz de Fora, Juiz de Fora, 2009.

OLIVEIRA, M. C. P.; OLIVEIRA, B. T. A.; DIAS, J. S.; MOURA, M. N.; SILVA, B. M.; SILVA, S. V. B. et al. Avaliação macroscópica da qualidade das nascentes do campus da Universidade Federal de Juiz de Fora. Revista Geografia, v. 3, n. 1, 2013.

ROCHA, C. H. B.; FREITAS, F. A.; SILVA, T. M. Alterações em variáveis limnológicas de manancial de Juiz de Fora devido ao uso da terra. Revista Brasileira de Engenharia Agrícola e Ambiental, v. 18, n. 4, p. 431-436, 2014. http://dx.doi.org/10.1590/S141543662014000400011

ROCHA, C. H. B.; COSTA, H. F. Variação temporal de parâmetros limnológicos em manancial de abastecimento em Juiz de Fora, MG. Revista Brasileira de Recursos Hídricos, v. 20, n. 2, p. 543-550, 2015. http://dx.doi.org/10.21168/rbrh.v20n2.p543-550

ROSA, B. F. J. V.; DE OLIVEIRA, V. C.; DA GAMA ALVES, R. Structure and spatial distribution of the Chironomidae community in mesohabitats in a first order stream at the Poço D'Anta Municipal Biological Reserve in Brazil. Journal of Insect Science, v. 11, n. 1, p. 1-13, 2011. http://dx.doi.org/10.1673/031.011.0136

SHEELA, A. M.; LETHA, J.; JOSEPH, S.; CHACKO, M.; SANAL KUMAR, S. P.; THOMAS, J. Water quality assessment of a tropical coastal lake system using multivariate cluster, principal component and factor analysis. Lakes \& Reservoirs: Research \& Management, v. 17, n. 2, p. 143-159, 2012. http://dx.doi.org/10. 1111/j.1440-1770.2012.00506.x

SOUSA, D. M. G.; LOBATO, E. Latossolos. Disponível em: http://www.agencia.cnptia. embrapa.br/Agencia16/AG01/arvore/AG01_96_10112005101956.html. Acesso em 13 mar. 2015. 
SURIANO, M. T.; FONSECA-GESSNER, A. A.; ROQUE, F. O.; FROEHLICH, C. G. Choice of macroinvertebrates metrics to evaluate stream conditions in Atlantic Forest, Brazil. Environmental Monitoring and Assessment, v. 175, p. 87-101, 2011. http://dx.doi.org/10.1007/s10661-010-1495-3

SILVEIRA, L. S. et al. Colonization by Chironomidae larvae in decomposition leaves of Eichhornia azurea in a lentic system in southeastern Brazil. Journal of Insect Science, v. 13, p. 1-13, 2013. http://dx.doi.org/10.1673/031.013.2001

SOARES, M. C. et al. Cyanobacterial equilibrium phases in a small tropical impoundment. Journal of Plankton Research, v. 31, p. 1331-8, 2009, http://dx.doi.org/ 10.1093/plankt/fbp079

UNIVERSIDADE FEDERAL DE JUIZ DE FORA - UFJF. Portal da Universidade Federal de Juiz de Fora. 2015. Disponível em: www.ufjf.br/portal/universidade/ufjf/dadosestatisticos/. Acesso em: 12 jul. 2015.

VON SPERLING, M. Introdução à qualidade das águas e ao tratamento de esgotos. 3. ed. Belo Horizonte: Imprensa Universitária da UFMF, 2005. 452 p.

WANG, X.; YIN, Z. Y. Using GIS to assess the relationship between land use and water quality at a watershed level. Environment International, v. 23, n. 1, p. 103-114, 1997. http://dx.doi.org/10.1016/S0160-4120(96)00081-5

WANTZEN, K.; MOL, J. Soil erosion from agriculture and mining: a threat to tropical stream ecosystems. Agriculture, v. 3, n. 4, p. 660-683, 2013. http://dx.doi.org/10.3390/ agriculture3040660

YU, D.; SHI, P.; LIU, Y.; XUN, B. Detecting land use-water quality relationships from the viewpoint of ecological restoration in an urban area. Ecological Engineering, v. 53, p. 205-216, 2013. http://dx.doi.org/10.1016/j.ecoleng.2012.12.045 